\title{
Methodological stumbling blocks
}

There are many barriers to the uptake of new methods in biological research. When methods cross disciplines, these can rise even higher.

Methods development in biology is not an end in itself. New methods must first enter the minds and then the laboratories of potential users, who often work in a different field than the developers. When uptake involves changing typical ways of thinking about how a biological question should be posed, it can take even longer for a method to be recognized as potentially valuable, let alone put to actual use. This is unfortunate because new tools can enable new and better research.

As an example of such a cross-disciplinary method, in a Perspective (p. 801), Fields and Fowler argue for the application of deep mutational scanning - in which deep sequencing monitors the phenotypic effects of mutating potentially every amino acid in a protein into every other amino acid - to the study of fundamental protein properties. The authors point out many challenges to this approach: not least among them is that it is essentially a genetic strategy (i.e., make many variants and examine their phenotypes) proposed to solve problems in the realm of classical biochemistry.

In a twist on a similar theme, Muir and colleagues (p. 834) use biochemical techniques in epigenetics. Their method relies on protein semisynthesis and ligation to generate barcoded nucleosome libraries, which they use to study the effect of defined post-translational histone modifications on the activity of proteins that 'read' chromatin. In still other recent work, researchers have used proximity-dependent biotinylation to map nuclear pore architecture; in an extended form, such a biochemical approach may well contribute to maps of subcellular organization, an area of inquiry classically thought of as cell biology.

The ability to recognize the value of an unconventional approach for solving a research problem can only be applauded. But there are other, more practical, barriers to interdisciplinary methods uptake.

A scientist must first become aware of a newly developed method, which is often not trivial. Methods development often happens in fields far from biology. Physicists develop new microscopic techniques, engineers make materials that biologists could use for cell culture experiments, chemists develop mass spectrometric approaches to solve protein structure. Of course, many methods developers either collaborate with biologists or have a biological research program themselves. In addition, the raison dêtre of a journal such as Nature Methods is to disseminate methodological developments across disciplines. But the size of the modern scientific enterprise is vast, and many potentially good methods may never reach the biologists who would benefit most from them.

How a method is presented also matters. To convince potential users of its value, a method must be demonstrated on the kind of data the users actually care about and not just 'toy' data that may appear designed to show a method in its best light. When alternative approaches exist, the performance of a new method should ideally be compared to theirs, although this is not always straightforward to do in an interpretable way. Paradoxically, very rapid methods development-far-red and near-infrared fluorophores or genome-editing tools, for instance, are published fast and furiously these days-may put off potential users from taking the plunge for fear of investing in a new tool that will be obsolete before they have even completed their first study.

Finally, the barriers of cost (such as for new equipment), time (for optimization) and the possible need to acquire new expertise will always remain. Periods of reduced resources, such as the one we are currently in, are likely to make researchers more conservative when it comes to adopting new approaches.

When a method's time has come, barriers fall remarkably quickly. Perhaps the most obvious recent example is genome editing with the nucleic acidguided CRISPR-Cas9 system. But CRISPR is relatively simple to implement, its path was smoothed by decades of experiments with other targeted editing platforms, and, critically, using CRISPR-Cas9 to make targeted genomic changes is a genetic approach applied to a genetic problem. It does not ask for a shift in thinking, unlike the use of mutagenesis screening for determining protein structure or of mass spectrometry for elucidating cellular organization.

Darwin credited his reading of Thomas Malthus, who wrote on overpopulation and the scarcity of resources from an economic perspective, with helping him to crystallize the theory of evolution by natural selection. It takes flexibility to put an old tool to new use or to take a fresh approach to an old problem. Scientists who can do so are clearly in august company. 